\title{
TRATAMENTO DAS ÁGUAS DO PROCESSAMENTO DOS FRUTOS DO CAFEEIRO EM FILTRO ANAERÓBIO SEGUIDO POR SISTEMA ALAGADO CONSTRUÍDO: II - REMOÇÃO DE NUTRIENTES E COMPOSTOS FENÓLICOS
}

\author{
RONALDO FIA ${ }^{1}$, ANTONIO T. DE MATOS ${ }^{2}$, TÚLIO F. LAMBERT ${ }^{3}$, \\ FÁTIMA R. L. FIA ${ }^{4}$, MATEUS P. DE MATOS
}

\begin{abstract}
RESUMO: Seis sistemas de tratamento foram compostos por três filtros anaeróbios com escoamento descendente, seguidos por seis sistemas alagados construídos (SACs), sendo avaliados, sob o ponto de vista operacional, no tratamento da água residuária do processamento dos frutos do cafeeiro (ARC). Os filtros foram confeccionados em PVC (1,5 $\mathrm{m}$ de altura e $0,35 \mathrm{~m}$ de diâmetro) e preenchidos com brita $\mathrm{n}^{\mathrm{o}}$ 2 e os SACs foram constituídos por caixas de madeira (1,5 m de comprimento, $0,4 \mathrm{~m}$ de altura e 0,5 m de largura), sendo impermeabilizados e preenchidos com brita "zero". A ARC teve o pH corrigido com cal até valores próximos a 7,0, e a concentração de nutrientes alterada, com a adição de fertilizantes, de forma a se obter uma relação DBO/N/P igual a 100/5/1. Em metade dos SACs, foi plantado o azevém (Lolium multiflorum Lam.), e na outra metade, aveia-preta (Avena strigosa Schreb). Como resultado, observou-se que as espécies vegetais cultivadas nos SACs influenciaram nas eficiências de remoção de fósforo pelos sistemas $\left(\mathrm{SAC}_{3}\right.$ e $\mathrm{SAC}_{4} ; \mathrm{SAC}_{5}$ e $\left.\mathrm{SAC}_{6}\right)$. Os sistemas que receberam as menores cargas de nutrientes e compostos fenólicos, via ARC, apresentaram as maiores eficiências de remoção destas variáveis, tendo sido alcançadas remoções de $40 \%$ do $\mathrm{N}_{\mathrm{T}}$ e $50 \%$ do $\mathrm{P}_{\mathrm{T}}$. A remoção de compostos fenólicos totais foi superior a $65 \%$ na maior parte dos sistemas. Desta forma, considera-se ser viável a aplicação de filtros anaeróbios seguidos por sistemas alagados construídos no tratamento da ARC.
\end{abstract}

PALAVRAS-CHAVE: tratamento anaeróbio, wetlands construídos, água residuária agroindustrial, compostos fenólicos, café.

\section{WASTEWATER TREATMENT OF COFFEE FRUIT PROCESSING IN ANAEROBIC FILTER SYSTEM FOLLOWED BY CONSTRUCTED WETLAND: II - REMOVAL OF NUTRIENTS AND PHENOLIC COMPOUNDS}

\begin{abstract}
Six treatment systems composed of three anaerobic filters with downflow followed by six constructed wetlands (SACs) have been operationally evaluated in the treatment of wastewater from coffee fruit processing (ARC). The filters were made of PVC (1.5 m high and 0.35 $\mathrm{m}$ in diameter) and filled with n. 2 crushed stone. SACs were constructed of wood boxes $(1.5 \mathrm{~m}$ long, $0.4 \mathrm{~m}$ high and $0.5 \mathrm{~m}$ wide) being sealed with an HDPE geomembrane and filled with "zero" crushed stone. The ARC had the $\mathrm{pH}$ adjusted with lime to values close to 7.0 and concentration of nutrients modified to have a relationship BOD/N/P equal to 100/5/1. In half of the SACs was planted ryegrass (Lolium multiflorum Lam.) and in the other half oat (Avena strigosa Schreb). As a result, it was observed that the plant species grown in the SACs have influenced the removal efficiencies of phosphorus by the systems $\left(\mathrm{SAC}_{3}\right.$ e $\mathrm{SAC}_{4} ; \mathrm{SAC}_{5}$ e $\left.\mathrm{SAC}_{6}\right)$. The systems that received the lowest loads of nutrients and phenolic compounds showed the highest removal efficiencies, reaching $40 \%$ for $\mathrm{N}_{\mathrm{T}}$ and $50 \%$ for $\mathrm{P}_{\mathrm{T}}$. The removal of total phenolic compounds was higher than $65 \%$ in most systems. Thus, it is considered to be feasible the application of anaerobic filter system followed by constructed wetland system for the treatment of ARC.
\end{abstract}

KEYWORDS: anaerobic treatment, constructed wetlands, agroindustrial wastewater, phenolic compounds, coffee.

\footnotetext{
${ }^{1}$ Eng $^{\mathrm{o}}$ Agrícola e Ambiental, Prof. Adjunto, Departamento de Engenharia, UFLA, ronaldofia@ deg.ufla.br.

${ }^{2}$ Eng $^{\mathrm{O}}$ Agrícola, Prof. Associado, Departamento de Engenharia Agrícola, UFV, atmatos@ufv.br.

${ }^{3}$ Graduando em Engenharia Agrícola, UFV, tulioflambert@ hotmail.com.

${ }^{4}$ Eng $^{\mathrm{a}}$ Agrícola, Profa. Adjunta, Departamento de Engenharia, UFLA, fatimarlf@deg.ufla.br.

${ }^{5}$ Graduando em Engenharia Agrícola, UFV, mateus.matos@ufv.br.

Recebido pelo Conselho Editorial em: 28-5-2010

Aprovado pelo Conselho Editorial em: 25-10-2010
} 


\section{INTRODUÇÃO}

A produção de cafés lavados e descascados/despolpados gera um produto de melhor qualidade de bebida, sendo uma exigência crescente dos mercados consumidores, além de proporcionar melhores preços ao produto e, consequentemente, maior lucratividade aos produtores.

No processo de lavagem dos frutos e retirada da casca (descascamento) e da mucilagem que reveste os grãos (despolpa), são gerados, em média, quatro litros de efluentes para cada litro de frutos processados (MATOS et al., 2006). Estes efluentes são referidos como as águas residuárias do processamento dos frutos do cafeeiro (ARC).

As características da ARC são altamente variáveis, dependendo das características dos frutos processados, se verdes, maduros ou secos, e do tipo de processamento utilizado, podendo, ainda, ser realizada a recirculação das águas. A ARC apresenta uma relação entre a matéria orgânica (DBO) e os nutrientes (N e P) considerada elevada, geralmente, superior a 100/5/1 (FIA 2008; LUIZ, 2007), fator que pode dificultar o tratamento biológico. Desta forma, faz-se necessária a adição de nutrientes, para que o desenvolvimento de comunidades microbianas não seja limitado, o que garante maiores eficiências na degradação da matéria orgânica (FIA et al., 2007). Além disso, a ARC apresenta valores de $\mathrm{pH}$ reduzidos, sendo necessária sua correção para favorecer a aplicação em sistemas de tratamento biológico. Quando se faz a recirculação da água no processamento, o volume gasto é reduzido (MATOS et al., 2007), no entanto, a ARC produzida apresenta maior concentração de matéria orgânica e compostos recalcitrantes, como os compostos fenólicos, dificultando o processo de tratamento.

Para o tratamento biológico da ARC, têm sido utilizados processos anaeróbios (FIA et al., 2010a; FIA, 2008; BRUNO \& OLIVEIRA, 2008), os quais demandam pós-tratamento para que sejam obtidas melhores eficiências na remoção de matéria orgânica, compostos fenólicos e nutrientes. Como pós-tratamento de efluentes anaeróbios, de forma geral, tem sido sugerida a aplicação em sistemas alagados construídos - SACs (CHERNICHARO, 2007), obtendo-se desempenho satisfatório no pós-tratamento da ARC (FIA, 2008).

Em geral, os SACs removem poluentes por meio de processos físicos, como precipitação, sedimentação, filtração e processos biogeoquímicos que reciclam e transformam alguns elementos químicos, como o carbono, o fósforo, o nitrogênio, entre outros. Nos SACs cultivados, o nitrogênio é mobilizado pelos processos físicos de sedimentação e suspensão de partículas, deposição a partir da atmosfera, difusão das formas dissolvidas, assimilação e translocação pelas plantas, volatilização da amônia, adsorção de nitrogênio solúvel pelo meio suporte, migração de organismos e acumulação resultante do decaimento da planta (KADLEC \& WALLACE, 2008).

Os principais mecanismos que removem $\mathrm{P}$ em alagados construídos incluem apenas sorção sobre substratos, armazenamento na biomassa, e a formação e acréscimo de turfa. O primeiro processo é, no entanto, saturável, o que significa que ele tem capacidade finita e, portanto, não pode contribuir, em longo prazo, na remoção de fósforo do sistema (KADLEC \& WALLACE, 2008). O potássio não se encontra associado à matéria orgânica e está incluído entre os nutrientes absorvidos pelos vegetais, a principal forma de remoção (BRASIL et al., 2005)

Diante dos resultados positivos obtidos por FIA (2008), no presente trabalho, objetivou-se estudar o desempenho de sistemas de tratamento compostos por filtros anaeróbios, seguidos de sistemas alagados construídos (SACs), submetidos a diferentes cargas de nutrientes e compostos fenólicos, no tratamento das águas residuárias do processamento dos frutos do cafeeiro.

\section{MATERIAL E MÉTODOS}

O experimento foi conduzido na Área de Pré-Processamento e Armazenamento de Produtos Agrícolas, do Departamento de Engenharia Agrícola da Universidade Federal de Viçosa, Viçosa-MG. A estrutura física foi constituída por três filtros anaeróbios, de escoamento descendente, construídos com segmentos de tubo de PVC, de 0,35 m de diâmetro e comprimento de $1,5 \mathrm{~m}$, com 
capacidade total de 139,5 L, que foram preenchidos com meio suporte (brita de granito-gnaisse $\mathrm{n}^{\mathrm{o}}$ 2), perfazendo-se colunas de 1,2 m de altura. Antes da aplicação da ARC, esses filtros operaram com esgoto doméstico durante um período de três meses (FIA et al., 2009).

Os efluentes dos três filtros anaeróbios $\left(\mathrm{F}_{1}, \mathrm{~F}_{2}\right.$ e $\left.\mathrm{F}_{3}\right)$ foram lançados, respectivamente, em seis SACs $\left(\mathrm{SAC}_{1}\right.$ e $\mathrm{SAC}_{2}, \mathrm{SAC}_{3}$ e $\mathrm{SAC}_{4}, \mathrm{SAC}_{5}$ e $\left.\mathrm{SAC}_{6}\right)$ de escoamento subsuperficial horizontal, construídos em escala-piloto, constituídos por caixas de madeira de pínus $(0,4 \mathrm{~m}$ de altura x 0,5 m de largura x 1,5 m de comprimento), impermeabilizadas por geomembrana de PEAD (polietileno de alta densidade), posicionadas sobre o solo, em declividade de $0,01 \mathrm{~m} \mathrm{~m}^{-1}$. Como meio suporte, utilizou-se brita $\mathrm{n}^{\mathrm{0}}$ "zero" (diâmetro D-60 = 7,0 $\mathrm{mm}$ e volume de vazios inicial de $0,491 \mathrm{~m}^{3} \mathrm{~m}^{-3}$ ). Os SACs foram preenchidos com a brita até a altura de $0,35 \mathrm{~m}$, deixando-se uma borda livre (não saturada) de $0,05 \mathrm{~m}$, já que o nível d'água foi mantido em $0,30 \mathrm{~m}$. Nos $\mathrm{SAC}_{1}, \mathrm{SAC}_{3}$ e $\mathrm{SAC}_{5}$ foi plantado o azevém (Lolium multiflorum), e nos $\mathrm{SAC}_{2}, \mathrm{SAC}_{4}$ e $\mathrm{SAC}_{6}$, a aveia-preta (Avena strigosa Schreb). Estas duas espécies, além de serem forrageiras de inverno, período no qual há produção da ARC, apresentaram bom desempenho agronômico quando cultivadas em rampas vegetadas para o tratamento da ARC por escoamento superficial (MATOS et al., 2005). O azevém foi plantado por meio de sementes $\left(30 \mathrm{~kg} \mathrm{ha}^{-1}\right)$, aplicadas a lanço sobre o leito de brita, e a aveia foi plantada a lanço $\left(80 \mathrm{~kg} \mathrm{ha}^{-1}\right)$, no entanto, devido à dificuldade na germinação, foi feito o transplantio aos 15 dias após a germinação $\left(60\right.$ plantas $\mathrm{m}^{-2}$ ). Após a semeadura, manteve-se o nível de água a 0,05 m abaixo da superfície do leito de brita, tendo as sementes permanecido umedecidas em decorrência da ascensão capilar da água no leito de brita.

A ARC foi proveniente do Sítio Jatobá, propriedade agrícola situada a $12 \mathrm{~km}$ da cidade de Viçosa, onde o consumo médio de água era de $2,5 \mathrm{~L} \mathrm{~L}^{-1}$ de grãos processados, pois havia recirculação da água no processo. Os grãos eram lavados e descascados/despolpados, tendo parte da polpa removida. Os filtros anaeróbios foram alimentados com ARC diluída, com correção de $\mathrm{pH}$ até aproximadamente 7,0, com cal hidratada, e com correção nutricional com ureia e superfosfato simples para se obter uma relação DBO/N/P de 100/5/1 (FIA et al., 2007) e, após 15 dias de implantação do sistema, iniciou-se a aplicação, dos efluentes gerados nos SACs. As características da ARC bruta antes da diluição e aplicação aos filtros e, posteriormente, aos SACs estão apresentadas na Tabela 1 .

TABELA 1. Valores médios das principais características da ARC bruta utilizada durante o período de operação do sistema. Average values of the principal characteristics of the untreated ARC used during the operation of the system.

\begin{tabular}{lccccccc}
\hline & $\mathrm{pH}$ & $\mathrm{DQO}$ & $\mathrm{DBO}$ & $\mathrm{F}_{\mathrm{T}}$ & $\mathrm{N}_{\mathrm{T}}$ & $\mathrm{P}_{\mathrm{T}}$ & $\mathrm{K}_{\mathrm{T}}$ \\
\hline ARC bruta & $3,44 \pm 0,16$ & $47.071 \pm 9.044$ & $13.471 \pm 3.999$ & $127 \pm 19$ & $228 \pm 18$ & $30 \pm 17$ & $686 \pm 56$ \\
\hline pH - potencial hidrogeniônico; $\mathrm{DQO}$ & - demanda química de oxigênio $\left(\mathrm{mg} \mathrm{L}^{-1}\right) ; \mathrm{DBO}$ - demanda bioquímica de oxigênio $\left(\mathrm{mg} \mathrm{L}^{-1}\right) ; \mathrm{N}_{\mathrm{T}}$ \\
- nitrogênio total $\left(\mathrm{mg} \mathrm{L}^{-1}\right) ; \mathrm{P}_{\mathrm{T}}$ - fósforo total $\left(\mathrm{mg} \mathrm{L}^{-1}\right) ; \mathrm{K}_{\mathrm{T}}$ & - potássio total $\left(\mathrm{mg} \mathrm{L}^{-1}\right) ; \mathrm{F}_{\mathrm{T}}$ - concentração de fenóis totais $\left(\mathrm{mg} \mathrm{L}^{-1}\right.$ de ácido \\
tânico).
\end{tabular}

O experimento foi monitorado durante 55 dias, compreendendo os meses de agosto e setembro, período no qual foram realizadas cinco amostragens, onde foram coletadas amostras afluentes e efluentes dos filtros, e efluentes dos SACs, para a avaliação do $\mathrm{pH}$; nitrogênio total (N) pelo método semimicro Kjeldahl, com adição de ácido salicílico, adaptado de JACKSON (1976); potássio total $(\mathrm{K})$, por fotometria de chama; fósforo total $(\mathrm{P})$, por espectrofotometria (APHA et al., 2005), todos obtidos após as amostras terem sido submetidas à digestão nítrico-perclórico; e compostos fenólicos totais (FOLIN \& CIOCALTEU, 1927).

Os valores médios e o desvio-padrão das características operacionais dos filtros anaeróbios e dos SACs estão apresentados na Tabela 2. 
TABELA 2. Condições operacionais utilizadas nos filtros anaeróbios e sistemas alagados construídos. Operational conditions of anaerobic filters and constructed wetlands.

\begin{tabular}{lcccccc}
\hline Variáveis & $\mathrm{TDH}$ & $\begin{array}{c}\mathrm{COV} \text { ou } \\
\mathrm{TCO}_{\mathrm{A}}\end{array}$ & $\mathrm{TC}_{\mathrm{N}}$ & $\mathrm{TC}_{\mathrm{P}}$ & $\mathrm{TC}_{\mathrm{K}}$ & $\mathrm{TC}_{\mathrm{FT}}$ \\
\hline $\mathrm{F}_{1}$ & $10,2 \pm 1,3$ & $1,91 \pm 0,29$ & $0,065 \pm 0,016$ & $0,008 \pm 0,004$ & $0,143 \pm 0,089$ & $0,028 \pm 0,016$ \\
$\mathrm{SAC}_{1}$ & $42,1 \pm 5,1$ & $653 \pm 470$ & $19,6 \pm 8,5$ & $2,1 \pm 1,4$ & $38,6 \pm 5,6$ & $5,6 \pm 1,5$ \\
$\mathrm{SAC}_{2}$ & $40,2 \pm 5,2$ & $686 \pm 447$ & $20,5 \pm 8,9$ & $2,2 \pm 1,5$ & $40,5 \pm 5,9$ & $5,9 \pm 1,6$ \\
$\mathrm{~F}_{2}$ & $10,4 \pm 1,5$ & $3,66 \pm 0,62$ & $0,075 \pm 0,018$ & $0,009 \pm 0,005$ & $0,206 \pm 0,089$ & $0,033 \pm 0,011$ \\
$\mathrm{SAC}_{3}$ & $42,1 \pm 5,4$ & $1.297 \pm 305$ & $23,3 \pm 6,8$ & $2,2 \pm 1,2$ & $56,2 \pm 6,5$ & $8,4 \pm 1,3$ \\
$\mathrm{SAC}_{4}$ & $39,3 \pm 4,8$ & $1.260 \pm 130$ & $24,8 \pm 7,3$ & $2,4 \pm 1,2$ & $60,0 \pm 6,9$ & $8,9 \pm 1,4$ \\
$\mathrm{~F}_{3}$ & $10,3 \pm 1,5$ & $4,89 \pm 1,12$ & $0,096 \pm 0,018$ & $0,011 \pm 0,005$ & $0,236 \pm 0,104$ & $0,042 \pm 0,018$ \\
$\mathrm{SAC}_{5}$ & $43,1 \pm 4,5$ & $1.394 \pm 426$ & $29,4 \pm 7,4$ & $2,9 \pm 1,5$ & $65,1 \pm 4,8$ & $9,3 \pm 1,8$ \\
SAC $_{6}$ & $42,6 \pm 3,6$ & $1.531 \pm 444$ & $29,6 \pm 7,5$ & $2,9 \pm 1,5$ & $65,4 \pm 4,8$ & $9,3 \pm 1,9$ \\
\hline
\end{tabular}

TDH - tempo de detenção hidráulica (h); COV - carga orgânica volumétrica aplicada aos filtros $\left(\mathrm{kg} \mathrm{m}^{-3} \mathrm{~d}^{-1}\right.$ de DQO); TCO $\mathrm{A}_{\mathrm{A}}$ t taxa de carga orgânica aplicada aos SACs $\left(\mathrm{kg} \mathrm{ha}^{-1} \mathrm{~d}^{-1} \mathrm{de} \mathrm{DQO}\right) ; \mathrm{TC}_{\mathrm{N}}$ - taxa de carga de nitrogênio; $\mathrm{TC}_{\mathrm{P}}$ - taxa de carga de fósforo; $\mathrm{TC}_{\mathrm{K}}$ - taxa de carga de potássio; $\mathrm{TC}_{\mathrm{FT}}$ - taxa de carga de compostos fenólicos. A taxa de carga teve como base o volume útil dos filtros $\left(\mathrm{kg} \mathrm{m}^{-3} \mathrm{~d}^{-1}\right)$ e a área superficial dos SACs $\left(\mathrm{kg} \mathrm{ha}^{-1} \mathrm{~d}^{-1}\right)$. Foram coletadas cinco amostras em cada fase para cálculo das taxas de carga; a Q e o TDH foram monitorados diariamente.

$\mathrm{O}$ experimento foi monitorado durante 55 dias, compreendendo os meses de agosto e setembro, período no qual foram realizadas cinco amostragens, onde foram coletadas amostras afluentes e efluentes dos filtros, e efluentes dos SACs, para a avaliação do pH; nitrogênio total (N) pelo método semimicro Kjeldahl, com adição de ácido salicílico, adaptado de JACKSON (1976); potássio total $(\mathrm{K})$, por fotometria de chama; fósforo total $(\mathrm{P})$, por espectrofotometria (APHA et al., 2005), todos obtidos após as amostras terem sido submetidas à digestão nítrico-perclórico; e compostos fenólicos totais (FOLIN \& CIOCALTEU, 1927).

Considerando-se os sistemas de tratamento ( $\mathrm{F}+\mathrm{SAC})$, as variáveis $\mathrm{N}_{\mathrm{T}}, \mathrm{P}_{\mathrm{T}}, \mathrm{K}_{\mathrm{T}}$ e os compostos fenólicos $\left(\mathrm{F}_{\mathrm{T}}\right)$ foram analisados estatisticamente no esquema fatorial 2 × 3 (duas espécies vegetais e três taxas de carga de nutrientes e compostos fenólicos), no delineamento em blocos casualizados (DBC), com cinco repetições (repetições no tempo). Procedeu-se à análise de variância, e as médias foram comparadas, utilizando-se do teste de Tukey, adotando-se o nível de 5\% de probabilidade.

\section{RESULTADOS E DISCUSSÃO}

O sistema operou sob temperatura ambiente, que variou de 13,3 a $23,2{ }^{\circ} \mathrm{C}$, e a temperatura média do período foi de $17,4 \pm 2,2{ }^{\circ} \mathrm{C}$, sendo esta faixa de temperatura considerada psicrofílica para os microrganismos. Com relação à ocorrência de chuvas, foi registrada durante a fase experimental uma lâmina de 138,8 mm, ocorrida ao longo do período de condução do experimento, a qual não influenciou nos resultados experimentais, por não ser coincidente com o período de amostragem.

Na Tabela 3, estão apresentados os valores médios de $\mathrm{pH}, \mathrm{N}_{\mathrm{T}}, \mathrm{P}_{\mathrm{T}}, \mathrm{K}_{\mathrm{T}}$ e $\mathrm{F}_{\mathrm{T}}$ afluentes $\left(\mathrm{C}_{1}, \mathrm{C}_{2} \mathrm{e}\right.$ $\left.\mathrm{C}_{3}\right)$ e efluentes dos filtros $\left(\mathrm{F}_{1}, \mathrm{~F}_{2} \mathrm{e} \mathrm{F}_{3}\right)$ e dos SACs $\left(\mathrm{SAC}_{1}, \mathrm{SAC}_{2}, \mathrm{SAC}_{3}, \mathrm{SAC}_{4}, \mathrm{SAC}_{5}\right.$ e $\left.\mathrm{SAC}_{6}\right)$.

Houve tendência de redução nos valores de $\mathrm{pH}$ nos efluentes dos filtros, quando comparados aos obtidos nos afluentes. No entanto, os valores mantiveram-se dentro da faixa considerada adequada para a digestão anaeróbia (CHERNICHARO, 2007).

As menores concentrações de $\mathrm{P}_{\mathrm{T}}$ e $\mathrm{F}_{\mathrm{T}}$ aplicadas neste trabalho, quando comparadas às aplicadas em outros trabalhos (FIA et al., 2010b; BRUNO \& OLIVEIRA, 2008), podem estar associadas à remoção parcial destas variáveis, proporcionadas pela cal, adicionada à ARC para correção do seu pH, tal como observado por TANAKA et al. (2007) e HSU et al. (2007). 
TABELA 3. Valores médios e desvio-padrão de $\mathrm{pH}$, nitrogênio total $\left(\mathrm{N}_{\mathrm{T}}\right)$, fósforo total $\left(\mathrm{P}_{\mathrm{T}}\right)$, potássio total $\left(\mathrm{K}_{\mathrm{T}}\right)$ e compostos fenólicos totais $\left(\mathrm{F}_{\mathrm{T}}\right)$, afluente $\left(\mathrm{C}_{1}, \mathrm{C}_{2}\right.$ e $\left.\mathrm{C}_{3}\right)$ e efluente dos filtros anaeróbios $\left(\mathrm{F}_{1}, \mathrm{~F}_{2}\right.$ e $\left.\mathrm{F}_{3}\right)$ e dos sistemas alagados construídos $\left(\mathrm{SAC}_{1}, \mathrm{SAC}_{2}\right.$, $\left.\mathrm{SAC}_{3}, \mathrm{SAC}_{4}, \mathrm{SAC}_{5}, \mathrm{SAC}_{6}\right)$. Mean values and standard deviation of $\mathbf{p H}$, total nitrogen $\left(N_{T}\right)$, total phosphorus $\left(P_{T}\right)$, total potassium $\left(K_{T}\right)$ and total phenolic compounds $\left(F_{T}\right)$ influent $\left(C_{1}, C_{2}\right.$ e $\left.C_{3}\right)$ and effluent of anaerobic filters $\left(F_{1}, F_{2} e\right.$ $\mathrm{F}_{3}$ ) and constructed wetlands $\left(\mathrm{SAC}_{1}, \mathrm{SAC}_{2}, \mathrm{SAC}_{3}, \mathrm{SAC}_{4}, \mathrm{SAC}_{5}, \mathrm{SAC}_{6}\right)$.

\begin{tabular}{lccccc}
\hline & $\mathrm{pH}$ & $\mathrm{N}_{\mathrm{T}}$ & $\mathrm{P}_{\mathrm{T}}$ & $\mathrm{K}_{\mathrm{T}}$ & $\mathrm{F}_{\mathrm{T}}$ \\
\hline $\mathrm{C}_{1}$ & $6,52 \pm 0,25$ & $27 \pm 7$ & $3,3 \pm 1,9$ & $60 \pm 37$ & $11,9 \pm 6,6$ \\
$\mathrm{~F}_{1}$ & $6,42 \pm 0,13$ & $22 \pm 9$ & $2,7 \pm 1,8$ & $43 \pm 6$ & $6,2 \pm 1,7$ \\
$\mathrm{SAC}_{1}$ & $6,91 \pm 0,23$ & $15 \pm 13$ & $1,4 \pm 0,7$ & $39 \pm 16$ & $3,1 \pm 1,9$ \\
$\mathrm{SAC}_{2}$ & $7,00 \pm 0,27$ & $17 \pm 11$ & $1,2 \pm 1,0$ & $39 \pm 11$ & $3,1 \pm 1,3$ \\
\hline $\mathrm{C}_{2}$ & $6,63 \pm 0,25$ & $32 \pm 8$ & $4,0 \pm 2,0$ & $88 \pm 38$ & $14,0 \pm 4,5$ \\
$\mathrm{~F}_{2}$ & $6,33 \pm 0,31$ & $26 \pm 8$ & $2,9 \pm 1,5$ & $62 \pm 7$ & $9,3 \pm 1,4$ \\
$\mathrm{SAC}_{3}$ & $7,04 \pm 0,26$ & $24 \pm 17$ & $1,5 \pm 0,7$ & $47 \pm 15$ & $5,3 \pm 1,8$ \\
$\mathrm{SAC}_{4}$ & $7,16 \pm 0,27$ & $22 \pm 16$ & $2,0 \pm 0,9$ & $60 \pm 24$ & $4,2 \pm 1,1$ \\
\hline $\mathrm{C}_{3}$ & $6,46 \pm 0,12$ & $40 \pm 8$ & $4,5 \pm 1,9$ & $99 \pm 44$ & $17,5 \pm 7,5$ \\
$\mathrm{~F}_{3}$ & $6,24 \pm 0,20$ & $33 \pm 8$ & $3,7 \pm 2,0$ & $74 \pm 5$ & $10,5 \pm 2,1$ \\
$\mathrm{SAC}_{5}$ & $7,06 \pm 0,31$ & $32 \pm 17$ & $2,2 \pm 1,0$ & $72 \pm 25$ & $6,5 \pm 0,9$ \\
$\mathrm{SAC}_{6}$ & $7,06 \pm 0,26$ & $33 \pm 21$ & $2,4 \pm 1,3$ & $74 \pm 18$ & $6,0 \pm 0,8$ \\
\hline
\end{tabular}

$\mathrm{N}_{\mathrm{T}}, \mathrm{P}_{\mathrm{T}}, \mathrm{K}_{\mathrm{T}}$ em mg L $\mathrm{m}^{-1} . \mathrm{F}_{\mathrm{T}}$ em $\mathrm{mg} \mathrm{L}^{-1}$ de ácido tânico. As cargas de $\mathrm{N}_{\mathrm{T}}, \mathrm{P}_{\mathrm{T}}, \mathrm{K}_{\mathrm{T}}$ e $\mathrm{F}_{\mathrm{T}}$ aplicadas em $\mathrm{F}_{1}$ foram de 0,$065 ; 0,008 ; 0,143 \mathrm{e}$ 0,028; em $\mathrm{F}_{2}, 0,075 ; 0,009 ; 0,206$ e 0,$033 ; \mathrm{em} \mathrm{F}_{3}, 0,096 ; 0,011 ; 0,236$ e $0,042 \mathrm{~kg} \mathrm{~m}^{-3} \mathrm{~d}^{-1}$. Enquanto o $\mathrm{SAC}_{1}$ recebeu 19,6;2,1;38,6 e 5,$6 ; \mathrm{SAC}_{2}, 20,5 ; 2,2 ; 40,5$ e 5,$9 ; \mathrm{SAC}_{3}, 23,3 ; 2,2 ; 56,2$ e 8,$4 ; \mathrm{SAC}_{4}, 24,8 ; 2,4 ; 60,0$ e 8,9; $\mathrm{SAC}_{5}, 29,4 ; 2,9 ; 65,1$ e 9,3, $\mathrm{SAC}_{6}, 29,6$; 2,$9 ; 65,4$ e $9,3 \mathrm{~kg} \mathrm{ha}^{-1} \mathrm{~d}^{-1}$, respectivamente.

\section{Avaliação dos filtros anaeróbios}

As variações na vazão afluente aos filtros (dados não apresentados) provocaram oscilações na estabilidade do sistema, o que dificultou a obtenção de eficiências de remoção constantes durante o experimento. Durante o período de monitoramento, as remoções médias de nutrientes $(\mathrm{N}, \mathrm{P}$ e $\mathrm{K})$ e compostos fenólicos foram semelhantes para os três filtros, não havendo diferença estatística entre elas $(\mathrm{P}<0,05)$ (Tabela 4).

TABELA 4. Valores médios da eficiência de remoção $(\%)$ de nitrogênio total $\left(\mathrm{N}_{\mathrm{T}}\right)$, fósforo total $\left(\mathrm{P}_{\mathrm{T}}\right)$, potássio total $\left(\mathrm{K}_{\mathrm{T}}\right)$ e compostos fenólicos totais $\left(\mathrm{F}_{\mathrm{T}}\right)$ durante o período de operação dos filtros anaeróbios $\left(\mathrm{F}_{1}, \mathrm{~F}_{2}\right.$ e $\left.\mathrm{F}_{3}\right)$. Average values of the removal efficiencies $(\%)$ of total nitrogen $\left(N_{T}\right)$, total phosphorus $\left(P_{T}\right)$, total potassium $\left(K_{T}\right)$ and total phenolic compounds $\left(F_{T}\right)$ during the operation of anaerobic filters $\left(F_{1}\right.$, $\left.\mathbf{F}_{2} \mathbf{e} \mathbf{F}_{3}\right)$.

\begin{tabular}{cccc}
\hline Variáveis & $\mathrm{F}_{1}$ & $\mathrm{~F}_{2}$ & $\mathrm{~F}_{3}$ \\
\hline $\mathrm{N}_{\mathrm{T}}$ & $22,8^{\mathrm{a}}$ & $27,9^{\mathrm{a}}$ & $41,3^{\mathrm{a}}$ \\
$\mathrm{P}_{\mathrm{T}}$ & $20,2^{\mathrm{a}}$ & $27,2^{\mathrm{a}}$ & $20,4^{\mathrm{a}}$ \\
$\mathrm{K}_{\mathrm{T}}$ & $14,4^{\mathrm{a}}$ & $21,6^{\mathrm{a}}$ & $15,7^{\mathrm{a}}$ \\
$\mathrm{F}_{\mathrm{T}}$ & $41,1^{\mathrm{a}}$ & $29,5^{\mathrm{a}}$ & $34,8^{\mathrm{a}}$ \\
\hline
\end{tabular}

Dentro de uma mesma linha, médias seguidas de mesma letra não diferem entre si, a $5 \%$ de probabilidade. As cargas de $\mathrm{N}_{\mathrm{T}}, \mathrm{P}_{\mathrm{T}}, \mathrm{K}_{\mathrm{T}} \mathrm{e}$ $\mathrm{F}_{\mathrm{T}}$ aplicadas em $\mathrm{F}_{1}$ foram de $0,0650,008 ; 0,143$ e 0,028 ; em $\mathrm{F}_{2}, 0,075 ; 0,009 ; 0,206$ e 0,033 ; em $\mathrm{F}_{3}, 0,096 ; 0,011 ; 0,236$ e 0,042 $\mathrm{kg} \mathrm{m}^{-3} \mathrm{~d}^{-1}$, respectivamente.

LUIZ (2007) avaliou reator anaeróbio de leito fixo, preenchido com brita, operando sob temperatura ambiente, e obteve eficiências de remoção de $\mathrm{N}_{\mathrm{T}}, \mathrm{P}_{\mathrm{T}}$ e $\mathrm{K}_{\mathrm{T}}$ de $23 ; 52$ e $18 \%$, ao aplicar concentrações de 29; 3 e $118 \mathrm{mg} \mathrm{L}^{-1}$ de $\mathrm{N}_{\mathrm{T}}, \mathrm{P}_{\mathrm{T}}$ e $\mathrm{K}_{\mathrm{T}}$, com $\mathrm{TDH}$ médio de 38 horas. Ao aumentar as concentrações aplicadas para $71 ; 8$ e $401 \mathrm{mg} \mathrm{L}^{-1}$ de $\mathrm{N}_{\mathrm{T}}, \mathrm{P}_{\mathrm{T}}$ e $\mathrm{K}_{\mathrm{T}}$, respectivamente, para o mesmo 
TDH, aumentou-se também a eficiência de remoção de $\mathrm{N}_{\mathrm{T}}$ para $34 \%$. No entanto, a remoção de $\mathrm{P}_{\mathrm{T}} \mathrm{e}$ $\mathrm{K}_{\mathrm{T}}$ foi reduzida para 16 e $5 \%$, respectivamente.

A remoção de $\mathrm{F}_{\mathrm{T}}$ variou de 36 a $44 \%$ para concentrações afluentes que variaram entre 20 e $43 \mathrm{mg} \mathrm{L}^{-1}$. PRADO \& CAMPOS (2008) obtiveram eficiências máximas de remoção de $\mathrm{N}_{\mathrm{T}}, \mathrm{P}_{\mathrm{T}} \mathrm{e}$ compostos fenólicos iguais a 35; 61 e 70\%, para concentrações que variaram de 15 a 35; 38 a 351 e 30 a $380 \mathrm{mg} \mathrm{L}^{-1}$ de $\mathrm{N}_{\mathrm{T}}, \mathrm{P}_{\mathrm{T}}$ e $\mathrm{F}_{\mathrm{T}}$, em reator UASB operado com TDH entre 8 e 70 horas, utilizado no tratamento das águas do processamento dos frutos do cafeeiro.

FIA (2008) obteve eficiências de remoção negativas para $\mathrm{N}_{\mathrm{T}}, \mathrm{P}_{\mathrm{T}}$ e $\mathrm{K}_{\mathrm{T}}$ durante a fase de adaptação de filtros anaeróbios utilizados no tratamento da $\mathrm{ARC}$, o que foi atribuído à liberação de nutrientes do lodo utilizado como inóculo. O autor verificou que, com o aumento da carga orgânica aplicada aos filtros e o consequente aumento nas concentrações de $\mathrm{N}_{\mathrm{T}}$ de 296 para $539 \mathrm{mg} \mathrm{L} \mathrm{L}^{-1}, \mathrm{P}_{\mathrm{T}}$ de 9 para $13 \mathrm{mg} \mathrm{L}^{-1}$ e $\mathrm{K}_{\mathrm{T}}$ de 199 para $424 \mathrm{mg} \mathrm{L}^{-1}$, mantendo-se a $\mathrm{TDH}$ em 35 horas, houve aumento na eficiência do sistema.

No entanto, os valores máximos obtidos na remoção de $\mathrm{N}_{\mathrm{T}}$ e $\mathrm{K}_{\mathrm{T}}$ foram de 8 e $7 \%$. No caso do $\mathrm{P}_{\mathrm{T}}$, foram obtidas eficiências relativamente maiores, alcançando $48 \%$ na menor concentração aplicada. CHERNICHARO (2007) destaca que a degradação anaeróbia apresenta remoção insatisfatória de nutrientes, necessitando de tratamentos complementares para remoção destes no efluente tratado.

A remoção de compostos fenólicos foi reduzida com o aumento na concentração aplicada de 30 para $65 \mathrm{mg} \mathrm{L}^{-1}$ de $\mathrm{F}_{\mathrm{T}}$; entretanto, em uma terceira etapa, com a redução da concentração de $\mathrm{F}_{\mathrm{T}}(6$ a $19 \mathrm{mg} \mathrm{L}^{-1}$ ) e a adaptação dos microrganismos à $\mathrm{ARC}$, houve aumento da eficiência do sistema na remoção de compostos fenólicos, tendo sido alcançados valores de $75 \%$ quando aplicada a menor carga.

BRUNO \& OLIVEIRA (2008) obtiveram eficiência média de $72 \%$ na remoção de compostos fenólicos, aplicando concentrações entre 80 e $97 \mathrm{mg} \mathrm{L}^{-1}$ de $\mathrm{F}_{\mathrm{T}}$ em reator UASB de dois estágios, utilizados no tratamento da ARC, operando com TDH de 144 horas.

Deve-se destacar que os valores afluentes obtidos na literatura foram superiores aos aplicados neste trabalho. Entretanto, as eficiências obtidas neste trabalho são comparáveis àquelas obtidas por FIA et al. (2010b) e foram superiores às obtidas por FIA (2008), provavelmente devido às maiores cargas aplicadas por esse autor.

\section{Avaliação dos sistemas alagados construídos}

As eficiências médias na remoção de nutrientes e $\mathrm{F}_{\mathrm{T}}$ nos SACs foram influenciadas pelas cargas aplicadas (Tabela 5). A remoção de $\mathrm{N}_{\mathrm{T}}$ foi maior para as menores cargas aplicadas deste nutriente e, entre os SACs que receberam as mesmas cargas orgânicas, obteve-se tendência de maiores eficiências médias naqueles cultivados com azevém $\left(\mathrm{SAC}_{1}, \mathrm{SAC}_{3}\right.$ e $\left.\mathrm{SAC}_{5}\right)$, espécie que apresentou maior desenvolvimento vegetativo (FIA et al., 2010c), apesar de não ter sido verificada diferença estatística $(\mathrm{P}<0,05)$. Fato semelhante foi observado para remoção de $\mathrm{P}$, com exceção do $\mathrm{SAC}_{2}$ e $\mathrm{SAC}_{4}$.

IAMCHATURAPATR et al. (2007) obtiveram entre 80 e $99 \%$ de remoção de $\mathrm{N}_{\mathrm{T}}$ e $\mathrm{P}_{\mathrm{T}}$ em SACs cultivados, e entre 40 e $78 \%$ nos SACs não cultivados, respectivamente, ao tratarem água residuária rica em nitrato $\left(20,3 \mathrm{mg} \mathrm{L}^{-1}\right)$ e fosfato $\left(20,1 \mathrm{mg} \mathrm{L}^{-1}\right)$ com $\mathrm{TDH}$ de 14 dias. 
TABELA 5. Valores médios da eficiência de remoção (\%) de nitrogênio total $\left(\mathrm{N}_{\mathrm{T}}\right)$, fósforo total $\left(\mathrm{P}_{\mathrm{T}}\right)$, potássio total $\left(\mathrm{K}_{\mathrm{T}}\right)$ e compostos fenólicos totais $\left(\mathrm{F}_{\mathrm{T}}\right)$ durante o período de operação dos sistemas alagados construídos $\left(\mathrm{SAC}_{1}, \mathrm{SAC}_{2}, \mathrm{SAC}_{3}, \mathrm{SAC}_{4}, \mathrm{SAC}_{5}\right.$, $\left.\mathrm{SAC}_{6}\right)$. Average values of the removal efficiencies $(\%)$ of total nitrogen $\left(\mathrm{N}_{\mathrm{T}}\right)$, total phosphorus $\left(\mathbf{P}_{\mathrm{T}}\right)$, total potassium $\left(\mathrm{K}_{\mathrm{T}}\right)$ and total phenolic compounds $\left(\mathrm{F}_{\mathrm{T}}\right)$ during the operation of constructed wetlands $\left(\mathrm{SAC}_{1}, \mathrm{SAC}_{2}, \mathrm{SAC}_{3}, \mathrm{SAC}_{4}, \mathrm{SAC}_{5}, \mathrm{SAC}_{6}\right)$.

\begin{tabular}{crrrrrr}
\hline Variáveis & $\mathrm{SAC}_{1}$ & $\mathrm{SAC}_{2}$ & $\mathrm{SAC}_{3}$ & $\mathrm{SAC}_{4}$ & $\mathrm{SAC}_{5}$ & $\mathrm{SAC}_{6}$ \\
\hline $\mathrm{N}_{\mathrm{T}}$ & $34,3^{\mathrm{a}}$ & $26,2^{\mathrm{a}}$ & $25,0^{\mathrm{a}}$ & $22,4^{\mathrm{a}}$ & $9,5^{\mathrm{b}}$ & $7,9^{\mathrm{b}}$ \\
$\mathrm{P}_{\mathrm{T}}$ & $39,5^{\mathrm{a}}$ & $50,0^{\mathrm{a}}$ & $57,6^{\mathrm{a}}$ & $21,2^{\mathrm{b}}$ & $37,3^{\mathrm{a}}$ & $28,2^{\mathrm{b}}$ \\
$\mathrm{K}_{\mathrm{T}}$ & $5,7^{\mathrm{a}}$ & $6,5^{\mathrm{a}}$ & $3,3^{\mathrm{a}}$ & $3,4^{\mathrm{a}}$ & $2,7^{\mathrm{a}}$ & $0,0^{\mathrm{a}}$ \\
$\mathrm{F}_{\mathrm{T}}$ & $52,9^{\mathrm{a}}$ & $50,7^{\mathrm{a}}$ & $45,8^{\mathrm{a}}$ & $53,4^{\mathrm{a}}$ & $35,6^{\mathrm{b}}$ & $41,8^{\mathrm{b}}$ \\
\hline
\end{tabular}

Dentro de uma mesma linha, médias seguidas de mesma letra não diferem entre si, a $5 \%$ de probabilidade. As cargas de $\mathrm{N}_{\mathrm{T}}, \mathrm{P}_{\mathrm{T}}, \mathrm{K}_{\mathrm{T}} \mathrm{e}$ $\mathrm{F}_{\mathrm{T}}$ aplicadas aos sistemas alagados foram: $\mathrm{SAC}_{1}, 19,6 ; 2,1 ; 38,6$ e 5,6; $\mathrm{SAC}_{2}, 20,5 ; 2,2 ; 40,5$ e 5,9; $\mathrm{SAC}_{3}, 23,3 ; 2,2 ; 56,2$ e 8,4; $\mathrm{SAC}_{4}$, 24,$8 ; 2,4 ; 60,0$ e 8,$9 ; \mathrm{SAC}_{5}, 29,4 ; 2,9 ; 65,1$ e $9,3, \mathrm{SAC}_{6}, 29,6 ; 2,9 ; 65,4$ e 9,3 $\mathrm{kg} \mathrm{ha}^{-1} \mathrm{~d}^{-1}$, respectivamente.

Ao avaliar o desempenho de SACs operados com águas residuárias do processamento da cana-de-açúcar com TDH de 60 e 120 horas, OLGUÍn et al. (2008) obtiveram eficiências de remoção de $\mathrm{N}_{\mathrm{T}}$ entre 73 e $76 \%$, para concentração média afluente de $26 \mathrm{mg} \mathrm{L}^{-1}$. Os mesmos autores obtiveram eficiências negativas para remoção de $\mathrm{P}_{\mathrm{T}}$ e $\mathrm{K}_{\mathrm{T}}$, sendo as concentrações médias afluentes iguais a 2 e $164 \mathrm{mg} \mathrm{L}^{-1}$. SOHSALAM et al. (2008) aplicaram, em sistemas alagados construídos, cargas de 58 a $292 \mathrm{~kg} \mathrm{ha}^{-1} \mathrm{~d}^{-1}$ de $\mathrm{N}_{\mathrm{T}}$ e de 28 a $50 \mathrm{~kg} \mathrm{ha}^{-1} \mathrm{~d}^{-1}$ de $\mathrm{P}_{\mathrm{T}}$; e obtiveram eficiências médias de 72 a $92 \%$ e 72 a $77 \%$, respectivamente, na remoção de $\mathrm{N}_{\mathrm{T}}$ e $\mathrm{P}_{\mathrm{T}}$ de águas residuárias do processamento de carne de frutos do mar. FIA (2008) obteve remoção de $\mathrm{N}_{\mathrm{T}}$ e $\mathrm{P}_{\mathrm{T}}$ da ordem de $40 \mathrm{e}$ $54 \%$ em SAC que recebeu 18,2 e 3,2 $\mathrm{kg} \mathrm{ha}^{-1} \mathrm{~d}^{-1}$ de $\mathrm{N}_{\mathrm{T}}$ e $\mathrm{P}_{\mathrm{T}}$ proveniente da ARC pré-tratada em filtros anaeróbios. GREENWAY (2005) obteve remoções de 85 e $21 \%$ do $\mathrm{N}_{\mathrm{T}}$ e do $\mathrm{P}_{\mathrm{T}}$, contidos em esgotos domésticos pré-tratados, ao aplicar concentrações de 6 e $8 \mathrm{mg} \mathrm{L}^{-1}$ de $\mathrm{N}_{\mathrm{T}}$ e $\mathrm{P}_{\mathrm{T}}$, respectivamente, com TDH de 264 horas, em SACs cultivados com Typha domingensis, Eleocharis sphaceolata, Schoenoplectus validus, Marsilia sp., Spirodela sp. e Ceratophyllum demersum. VYMAZAL (2007), em uma revisão sobre remoção de nutrientes em SACs, afirma que a remoção de nitrogênio e fósforo varia de 40 a $55 \%$ e de 40 a $60 \%$, para cargas entre 6 e $17 \mathrm{~kg} \mathrm{ha}^{-1} \mathrm{~d}^{-1}$ e 1,2 e $2,1 \mathrm{~kg} \mathrm{ha}^{-1} \mathrm{~d}^{-1}$, respectivamente.

Baseando-se nos valores reportados por VYMAZAL (2007) e nas cargas de nutrientes aplicadas neste trabalho, entende-se que as eficiências de remoção de $\mathrm{N}_{\mathrm{T}}$ podem ser consideradas satisfatórias, nos $\mathrm{SAC}_{1}, \mathrm{SAC}_{2}, \mathrm{SAC}_{3}$ e $\mathrm{SAC}_{4}$, e as de $\mathrm{P}_{\mathrm{T}}$, nos $\mathrm{SAC}_{1}, \mathrm{SAC}_{2}$ e $\mathrm{SAC}_{3}$.

$\mathrm{O} \mathrm{K}_{\mathrm{T}}$ permanece em solução, não se associando aos compostos orgânicos, o que torna a eficiência de remoção nos SACs totalmente dependente da absorção vegetal. BRASIL et al. (2005) obtiveram eficiências médias de remoção de $\mathrm{K}_{\mathrm{T}}$ que variaram de 23 a 52\%; entretanto estes autores aplicaram taxas que variaram de 2 a $11 \mathrm{~kg} \mathrm{ha}^{-1} \mathrm{~d}^{-1}$ de $\mathrm{K}_{\mathrm{T}}$. MATOS et al. (2010) obtiveram eficiências de remoção que variaram de 29 a 46\%, para uma taxa de aplicação média de $36 \mathrm{~kg} \mathrm{ha}^{-1} \mathrm{dia}^{-1}$ de $\mathrm{K}_{\mathrm{T}}$. Ambos os autores aplicaram cargas de $\mathrm{K}_{\mathrm{T}}$ inferiores às aplicadas neste trabalho, que foi de 38,6 a $65,4 \mathrm{~kg} \mathrm{ha}^{-1} \mathrm{~d}^{-1}$ de $\mathrm{K}_{\mathrm{T}}$. FIA (2008) obteve eficiências relativamente baixas na remoção de $\mathrm{K}_{\mathrm{T}}$ (até $14 \%$ ) ao serem aplicadas cargas acima de $100 \mathrm{~kg} \mathrm{ha}^{-1} \mathrm{~d}^{-1}$ de $\mathrm{K}_{\mathrm{T}} \mathrm{em}$ SACs, no pós-tratamento da ARC em filtros anaeróbios. Torna-se importante, no entanto, ressaltar que, com o desenvolvimento das plantas, há aumento na evapotranspiração e consequente redução na vazão efluente dos SACs, proporcionando aumento na concentração de sais no meio, fenômeno que, provavelmente, mais influenciou no aumento da concentração efluente de potássio.

A remoção de $\mathrm{F}_{\mathrm{T}}$ também seguiu a tendência de redução com o aumento da carga aplicada, sem diferença estatística entre os SACs que receberam a mesma carga orgânica. GRISMER et al. (2003), no tratamento de águas residuárias da produção de vinho, obtiveram remoções de 48 a $78 \%$ de $\mathrm{F}_{\mathrm{T}}$, utilizando sistemas alagados construídos. Os autores aplicaram taxas médias de $17 \mathrm{~kg} \mathrm{ha}^{-1} \mathrm{~d}^{-1}$ de $\mathrm{F}_{\mathrm{T}}$ (estimada), quantificados como taninos, sendo o TDH de 132 horas. FIA (2008) aplicou em 
SACs, utilizados no pós-tratamento de ARC, em filtros anaeróbios, cargas que variaram de 4,6 a $6,7 \mathrm{~kg} \mathrm{ha}^{-1} \mathrm{~d}^{-1}$ de $\mathrm{F}_{\mathrm{T}}$, com TDH de 58 horas, e obteve eficiências de remoção entre 32 e $65 \%$. Apesar de GRISMER et al. (2003) terem aplicado maior carga de $\mathrm{F}_{\mathrm{T}}$ comparadas àquelas aplicadas por FIA (2008) e às aplicadas neste trabalho, o TDH dos SACs operados por GRISMER et al. (2003) foi cerca de três vezes maior que o aplicado neste trabalho. Assim, pode considerar-se que as eficiências de remoção de $\mathrm{F}_{\mathrm{T}}$ obtidas nos $\mathrm{SAC}_{1}, \mathrm{SAC}_{2}, \mathrm{SAC}_{3}$ e $\mathrm{SAC}_{4}$ estão satisfatórias, em função do reduzido TDH aplicado no tratamento da ARC.

\section{Avaliação global do sistema de tratamento}

A eficiência global do sistema (F+SAC), apresentada na Tabela 6, pode ser considerada satisfatória na remoção de $\mathrm{N}_{\mathrm{T}}$ e $\mathrm{P}_{\mathrm{T}}$, principalmente naqueles sistemas que receberam as menores cargas de $\mathrm{N}_{\mathrm{T}}\left(\mathrm{F}_{1}+\mathrm{SAC}_{1}\right.$ e $\left.\mathrm{F}_{1}+\mathrm{SAC}_{2}\right)$ e de $\mathrm{P}_{\mathrm{T}}\left(\mathrm{F}_{1}+\mathrm{SAC}_{1}, \mathrm{~F}_{1}+\mathrm{SAC}_{2}\right.$ e $\left.\mathrm{F}_{2}+\mathrm{SAC}_{3}\right)$. Fato semelhante também foi observado em relação à remoção de $\mathrm{F}_{\mathrm{T}}$. A eficiência na remoção de $\mathrm{K}_{\mathrm{T}}$ foi reduzida, tal como seria de se esperar e como observado na literatura.

TABELA 6. Valores médios da eficiência global de remoção (\%) de nitrogênio total $\left(\mathrm{N}_{\mathrm{T}}\right)$, fósforo total $\left(\mathrm{P}_{\mathrm{T}}\right)$, potássio total $\left(\mathrm{K}_{\mathrm{T}}\right)$ e compostos fenólicos totais $\left(\mathrm{F}_{\mathrm{T}}\right)$ durante o período de operação dos sistemas compostos por F+SAC. Average values of overall efficiency of removal $(\%)$ of total nitrogen $\left(\mathrm{N}_{\mathrm{T}}\right)$, total phosphorus $\left(\mathrm{P}_{\mathrm{T}}\right)$, total potassium $\left(K_{T}\right)$ and total phenolic compounds $\left(F_{T}\right)$ during the operation of anaerobic filter+constructed wetland system $(\mathrm{F}+\mathrm{SAC})$.

\begin{tabular}{ccccccc}
\hline Variáveis & $\mathrm{F}_{1}+\mathrm{SAC}_{1}$ & $\mathrm{~F}_{1}+\mathrm{SAC}_{2}$ & $\mathrm{~F}_{2}+\mathrm{SAC}_{3}$ & $\mathrm{~F}_{2}+\mathrm{SAC}_{4}$ & $\mathrm{~F}_{3}+\mathrm{SAC}_{5}$ & $\mathrm{~F}_{3}+\mathrm{SAC}_{6}$ \\
\hline $\mathrm{N}_{\mathrm{T}}$ & $47,7^{\mathrm{a}}$ & $42,0^{\mathrm{a}}$ & $33,3^{\mathrm{b}}$ & $30,7^{\mathrm{b}}$ & $23,8^{\mathrm{c}}$ & $22,0^{\mathrm{c}}$ \\
$\mathrm{P}_{\mathrm{T}}$ & $53,9^{\mathrm{a}}$ & $64,1^{\mathrm{a}}$ & $69,6^{\mathrm{a}}$ & $43,1^{\mathrm{b}}$ & $49,5^{\mathrm{a}}$ & $42,9^{\mathrm{b}}$ \\
$\mathrm{K}_{\mathrm{T}}$ & $11,2^{\mathrm{a}}$ & $14,6^{\mathrm{a}}$ & $14,1^{\mathrm{a}}$ & $19,3^{\mathrm{a}}$ & $16,6^{\mathrm{a}}$ & $13,0^{\mathrm{a}}$ \\
$\mathrm{F}_{\mathrm{T}}$ & $73,1^{\mathrm{a}}$ & $71,0^{\mathrm{a}}$ & $65,0^{\mathrm{a}}$ & $69,2^{\mathrm{a}}$ & $57,2^{\mathrm{b}}$ & $62,0^{\mathrm{b}}$ \\
\hline
\end{tabular}

Dentro de uma mesma linha, médias seguidas de mesma letra não diferem entre si, a $5 \%$ de probabilidade. As cargas de $\mathrm{N}_{\mathrm{T}}, \mathrm{P}_{\mathrm{T}}, \mathrm{K}_{\mathrm{T}} \mathrm{e}$ $\mathrm{F}_{\mathrm{T}}$ aplicadas em $\mathrm{F}_{1}$ foram de 0,$065 ; 0,008 ; 0,143$ e 0,$028 ;$ em F $_{2}, 0,075 ; 0,009 ; 0,206$ e 0,$033 ;$ em $\mathrm{F}_{3}, 0,096 ; 0,011 ; 0,236$ e $0,042 \mathrm{~kg}$ $\mathrm{m}^{-3} \mathrm{~d}^{-1}$. Enquanto o $\mathrm{SAC}_{1}$ recebeu 19,$6 ; 2,1 ; 38,6$ e 5,$6 ; \mathrm{SAC}_{2}, 20,5 ; 2,2 ; 40,5$ e 5,$9 ; \mathrm{SAC}_{3}, 23,3 ; 2,2 ; 56,2$ e 8,$4 ; \mathrm{SAC}_{4}, 24,8 ; 2,4$; 60,0 e 8,$9 ; \mathrm{SAC}_{5}, 29,4 ; 2,9 ; 65,1$ e $9,3, \mathrm{SAC}_{6}, 29,6 ; 2,9 ; 65,4$ e $9,3 \mathrm{~kg} \mathrm{ha}^{-1} \mathrm{~d}^{-1}$, respectivamente.

Diante dos resultados obtidos, verifica-se que os efluentes dos sistemas de tratamento $\left(\mathrm{F}_{2}+\mathrm{SAC}_{3}, \mathrm{~F}_{2}+\mathrm{SAC}_{4}, \mathrm{~F}_{3}+\mathrm{SAC}_{5}\right.$ e $\left.\mathrm{F}_{3}+\mathrm{SAC}_{6}\right)$ apresentam concentrações de nitrogênio superiores à estabelecida na Resolução COPAM/CERH n ${ }^{\circ} 01$ de 2008 para a disposição final em cursos de água, que é de $20 \mathrm{mg} \mathrm{L}^{-1}$ para o nitrogênio amoniacal total. Apesar de a forma de nitrogênio determinada neste trabalho ser diferente da forma estabelecida como padrão de lançamento, acredita-se que grande parte do nitrogênio total, determinada neste trabalho, seja formada pelo nitrogênio amoniacal, em função da degradação no nitrogênio orgânico e, provavelmente, em função das baixas concentrações de oxigênio dissolvido no meio devido à aplicação das elevadas cargas orgânicas, não resultando na conversão de formas nítricas. Desta forma, com o tratamento da ARC, há remoção de partículas sólidas, parte da matéria orgânica e dos $\mathrm{F}_{\mathrm{T}}$, sabidamente tóxicos às plantas, quando aplicado em excesso no solo (EL HADRAMI et al., 2004), podendo ser utilizada, como fonte de nutrientes, em processos de fertirrigação.

Em função das maiores concentrações de $\mathrm{K}_{\mathrm{T}}$ presentes na $\mathrm{ARC}$ e diante dos resultados obtidos neste trabalho, recomenda-se que a carga máxima a ser utilizada no sistema $\mathrm{F}+\mathrm{SAC}$ não ultrapasse $0,150 \mathrm{~kg} \mathrm{~m}^{-3} \mathrm{~d}^{-1}$ de $\mathrm{K}_{\mathrm{T}}$ e $0,040 \mathrm{~kg} \mathrm{~m}^{-3} \mathrm{~d}^{-1}$ de $\mathrm{F}_{\mathrm{T}}$ nos filtros anaeróbios, e $40 \mathrm{~kg} \mathrm{ha}^{-1} \mathrm{~d}^{-1} \mathrm{de}$ $\mathrm{K}_{\mathrm{T}}$ e $9 \mathrm{~kg} \mathrm{ha}^{-1} \mathrm{~d}^{-1}$ de $\mathrm{F}_{\mathrm{T}}$ nos SACs. As maiores eficiências de remoção de matéria orgânica obtidas por FIA (2008), ao aplicar ARC em filtros, anaeróbios seguidos por SACs, foram conseguidas ao aplicarem valores semelhantes de $\mathrm{K}_{\mathrm{T}}\left(60 \mathrm{~kg} \mathrm{ha}^{-1} \mathrm{~d}^{-1}\right)$ e $\mathrm{F}_{\mathrm{T}}\left(10 \mathrm{~kg} \mathrm{ha}^{-1} \mathrm{~d}^{-1}\right)$ nos SACs. Entretanto, nos filtros as taxas aplicadas foram inferiores a $0,080 \mathrm{~kg} \mathrm{~m}^{-3} \mathrm{~d}^{-1}$ de $\mathrm{K}_{\mathrm{T}}$ e $0,003 \mathrm{~kg} \mathrm{~m}^{-3} \mathrm{~d}^{-1}$ de $\mathrm{F}_{\mathrm{T}}$, proporcionando maiores eficiências, quando comparadas às obtidas neste trabalho. 
Para o tratamento da ARC em SACs, há necessidade de mais estudos e a determinação das variáveis de maior importância no dimensionamento dos sistemas. No entanto, os dados até aqui obtidos indicam a possibilidade da aplicação de sistemas conjugados formados por filtros anaeróbios e sistemas alagados construídos no tratamento da ARC para remoção de nutrientes e compostos fenólicos.

\title{
CONCLUSÕES
}

Os sistemas de tratamento compostos por filtros anaeróbios, seguidos de sistemas alagados construídos que receberam as menores cargas de nutrientes $\left(\mathrm{N}_{\mathrm{T}}, \mathrm{P}_{\mathrm{T}}\right.$ e $\mathrm{K}_{\mathrm{T}}$ aplicados em $\mathrm{F}_{1}, 0,065$, 0,008 e $0,143 \mathrm{~kg} \mathrm{~m}^{-3} \mathrm{~d}^{-1}$; no $\mathrm{SAC}_{1}, 19,6,2,1$ e $38,6 \mathrm{~kg} \mathrm{ha}^{-1} \mathrm{~d}^{-1}$; e no $\mathrm{SAC}_{2}, 20,5,2,2$ e $40,5 \mathrm{~kg} \mathrm{ha}^{-1} \mathrm{~d}^{-1}$, respectivamente) e compostos fenólicos $\left(\mathrm{F}_{\mathrm{T}}\right.$ aplicados em $\mathrm{F}_{1}, 0,028 \mathrm{~kg} \mathrm{~m}^{-3} \mathrm{~d}^{-1}$; no $\mathrm{SAC}_{1}, 5,6 \mathrm{~kg} \mathrm{ha}^{-1} \mathrm{~d}^{-1}$; e no $\mathrm{SAC}_{2}, 5,9 \mathrm{~kg} \mathrm{ha}^{-1} \mathrm{~d}^{-1}$, respectivamente), apresentaram as maiores eficiências na remoção desses poluentes. As espécies vegetais cultivadas nos SACs influenciaram nas eficiências de remoção de $\mathrm{P}_{\mathrm{T}}$ pelos sistemas $\left(\mathrm{SAC}_{3}\right.$ e $\mathrm{SAC}_{4} ; \mathrm{SAC}_{5}$ e $\left.\mathrm{SAC}_{6}\right)$. Quando da aplicação das menores cargas, a remoção do $\mathrm{N}_{\mathrm{T}}$ foi de $40 \%$ nos sistemas $\mathrm{F}_{1}+\mathrm{SAC}_{1}$ e $\mathrm{F}_{1}+\mathrm{SAC}_{2}$ e de $50 \%$ do $\mathrm{P}_{\mathrm{T}}$ nos sistemas $\mathrm{F}_{1}+\mathrm{SAC}_{1}, \mathrm{~F}_{1}+\mathrm{SAC}_{2}$ e $\mathrm{F}_{2}+\mathrm{SAC}_{3}$. A remoção de compostos fenólicos totais foi superior a $65 \%$ nos sistemas $\mathrm{F}_{1}+\mathrm{SAC}_{1}, \mathrm{~F}_{1}+\mathrm{SAC}_{2}, \mathrm{~F}_{2}+\mathrm{SAC}_{3}$ e $\mathrm{F}_{2}+\mathrm{SAC}_{4}$, indicando a viabilidade da aplicação do sistema $\mathrm{F}+\mathrm{SAC}$ no tratamento das águas residuárias do processamento dos frutos do cafeeiro.

\section{AGRADECIMENTOS}

À FAPEMIG (Bolsa de Pós-Doutorado) e ao CNPq, pelo apoio financeiro e bolsa concedidos.

\section{REFERÊNCIAS}

\begin{abstract}
APHA. AMERICAN PUBLIC HEALTH ASSOCIATION; AWWA. AMERICAN WATER WORKS ASSOCIATION; WEF. WATER ENVIRONMENT FEDERATION. Standard methods for the examination of water and wastewater. 21th. ed. Washington, 2005.
\end{abstract}

BRASIL, M.S.; MATOS, A.T.; SOARES; A.A.; FERREIRA, P.A. Qualidade de efluente de sistemas alagados construídos, utilizados no tratamento de esgoto doméstico. Revista Brasileira de Engenharia Agrícola e Ambiental, Campina Grande, v.9, p.133-137, 2005.

BRUNO, M.; OLIVEIRA, R.A. Tratamento anaeróbio de águas residuárias do beneficiamento de café por via úmida em reatores UASB, em dois estágios. Engenharia Agrícola, Jaboticabal, v.28, n.2, p.364-377, 2008.

CHERNICHARO, C.A.L. Reatores anaeróbios. 2.ed. Belo Horizonte: Departamento de Engenharia Sanitária e Ambiental, 2007. 380 p. (Princípios do tratamento biológico de águas residuárias, v.5).

COPAM. Conselho Estadual de Política Ambiental; CERH. Conselho Estadual de Recursos Hídricos. Resolução Conjunta COPAM/CERH n ${ }^{\circ} 1$ de 2008: dispõe sobre a classificação dos corpos de água e diretrizes ambientais para o seu enquadramento, bem como estabelece as condições e padrões de lançamento de efluentes, e dá outras providências. Disponível em: <http://www.siam.mg.gov.br>. Acesso em: 18 jun. 2008.

EL HADRAMI, A.; BELAQZIZ, M.; EL HASSNI, M.; HANIFI, S.; ABBAD, A.; CAPASSO, R.; GIANFREDA, L.; EL HADRAMI, I. Physico-chemical characterization and effects of olive oil mill wastewaters fertirrigation on the growth of some mediterranean crops. Agronomy Journal, Madison, v.3, n.4, p.247-254, 2004.

FIA, R. Desempenho de sistemas alagados construídos no tratamento de águas residuárias da lavagem e descascamento/despolpa dos frutos do cafeeiro. 2008. $181 \mathrm{f}$. Tese (Doutorado em Engenharia Agrícola) - Universidade Federal de Viçosa, Viçosa - MG, 2008. 
FIA, R.; SCHUERY, F.C.; MATOS, A.T.; FERRES, G.C.; OLIVEIRA, T.F. Avaliação comparativa entre filtros anaeróbios com escoamento ascendente e descendente afogado. In: CONGRESSO BRASILEIRO DE ENGENHARIA AGRÍCOLA, 38., 2009, Juazeiro/Petrolina. Anais... Juazeiro/Petrolina: Associação Brasileira de Engenharia Agrícola, 2009. 1 CD-ROM.

FIA, F.R.L.; BORGES, A.C.; MATOS, A.T.; DUARTE, I.C.S.; FIA, R.; CAMPOS, L.C. Development of biofilm in anaerobic reactors treating wastewater from coffee grain processing. Revista Brasileira de Engenharia Agrícola e Ambiental, Campina Grande, v.14, n.2, p.210-217, 2010a.

FIA, F.R.L.; MATOS, A.T.; BORGES, A.C.; MOREIRA, D.A.; FIA, R.; EUSTÁQUIO JÚNIOR, V. Remoção de compostos fenólicos em reatores anaeróbios de leito fixo com diferentes materiais suporte. Revista Brasileira de Engenharia Agrícola e Ambiental, Campina Grande, v.14, n.10, p.1.079-1.086, 2010b.

FIA, R.; MATOS, A.T.; FIA, F.R.L.; MATOS, M.P.; LAMBERT, T.F.; NASCIMENTO, F.S. Desempenho de forrageiras em sistemas alagados de tratamento de águas residuárias do processamento do café. Revista Brasileira de Engenharia Agrícola e Ambiental, Campina Grande, v.14, n.8, p.842-847, 2010c.

FIA, R.; MATOS, A.T.; LUIZ, F.A.R.; PEREIRA, P.A. Coeficientes de degradação da matéria orgânica de água residuária da lavagem e descascamento dos frutos do cafeeiro em condições anóxica e aeróbia. Engenharia na Agricultura, Viçosa-MG, v.15, n.1, p.45-54, 2007.

FOLIN, O.; CIOCALTEU, V. On tyrosine and tryptophane determinations in proteins. The Journal of Biological Chemistry, Bethesda, v.73, n.2, p.627-650, 1927.

GREENWAY, M. The role of constructed wetlands in secondary effluent treatment and water reuse in subtropical and arid Australia. Ecological Engineering, Oxford, v.25, p.501-509, 2005.

GRISMER, M.E.; CARR, M.A.; SHEPHERD, H.L. Evaluation of constructed wetland treatment performance for winery wastewater. Water Environmental Research, Alexandria, v.75, n.5, p.412421, 2003.

HSU, Y-C.; CHEN, J-H.; YANG, H-C. Calcium enhanced COD removal for the ozonation of phenol solution. Water Research, New York, v.41, n.1, p.71-78, 2007.

IAMCHATURAPATR, J.; YI, S.W.; RHEE, J.S. Nutrient removals by 21 aquatic plants for vertical free surface-flow (VFS) constructed wetland. Ecological Engineering, Oxford, v.29, p.287-293, 2007.

JACKSON, M.L. Análisis químico de suelos. Trad. de J.B. Martínez. 3.ed. Barcelona: Ediciones Omega, 1976. $662 \mathrm{p}$.

KADLEC, R.H.; WALLACE, S.D. Treatment wetlands. 2.ed. Boca Raton: CRC Press, 2008. $1.016 \mathrm{p}$.

LUIZ, F.A.R. Desempenho de reatores anaeróbios de leito fixo no tratamento de águas residuárias da lavagem e descascamento/despolpa dos frutos do cafeeiro. 2007. 132 f. Dissertação (Mestrado em Engenharia Agrícola) - Universidade Federal de Viçosa, Viçosa - MG, 2007.

MATOS, A.T.; CABANELLAS, C.F.G.; CECON, P.R.; BRASIL, M.S.; MUDADO, C.S. Efeito da concentração de coagulantes e do $\mathrm{pH}$ da solução na turbidez da água, em recirculação, utilizada no processamento dos frutos do cafeeiro. Engenharia Agrícola, Jaboticabal, v.27, n.2, p.544-551, 2007.

MATOS, A.T.; FREITAS, W.S.; LO MONACO, P.A.V. Eficiência de sistemas alagados construídos na remoção de poluentes de águas residuárias da suinocultura. Revista Ambiente e Água, v.5, n.2, p.119-132, 2010. 
MATOS, A.T.; MAGALHÃES, M.A.; FUKUNAGA, D.C. Remoção de sólidos em suspensão na água residuária da despolpa de frutos do cafeeiro em filtros constituídos por pergaminho de grãos de café submetido a compressões. Engenharia Agrícola, Jaboticabal, v.26, n.2, p.610-616, 2006.

MATOS, A.T.; PINTO, A.B.; PEREIRA, O.G.; BARROS, F.M. Extração de nutrientes por forrageiras cultivadas com águas residuárias do beneficiamento de frutos do cafeeiro. Revista Ceres, Viçosa-MG, v.52, n.303, p.675-688, 2005.

OLGUÍN, E.J.; SÁNCHEZ-GALVÁN, G.; GONZÁ LEZ-PORTELA, R.E.; LÓPEZ-VELA, M. Constructed wetland mesocosms for the treatment of diluted sugarcane molasses stillage from ethanol production using Pontederia sagittata. Water Research, New York, v.42, p.3.659-3.666, 2008.

PRADO, M.A.C.; CAMPOS, C.M.M. Produção de biogás no tratamento dos efluentes líquidos do processamento de Coffea arabica L. em reator anaeróbio UASB para o potencial aproveitamento na secagem do café. Ciência e Agrotecnologia, Lavras-MG, v.32, n.3, p.938-947, 2008.

SOHSALAM, P.; ENGLANDE, A.J.; SIRIANUNTAPIBOON, S. Seafood wastewater treatment in constructed wetland: Tropical case. Bioresource Technology, Essex, v.99, p.1.218-1.224, 2008.

TANAKA, Y.; YATAGAI, A.; MASUJIMA, H.; WAKI, M.; YOKOYAMA, H. Autotrophic denitrification and chemical phosphate removal of agro-industrial wastewater by filtration with granular medium. Bioresource Technology, v.98, p.787-791, 2007.

VYMAZAL, J. Removal of nutrients in various types of constructed wetlands. Science of the Total Environment, Amsterdan, v.380, n.1, p.48-65, 2007. 